\title{
Application Of Signed Graph In Decision Making
}

\author{
Debanjan Banerjee $^{1}$, Anita Pal ${ }^{2}$ \\ ${ }^{1}$ Department of Mathematics, \\ NIT Durgapur, 713209, West Bengal, India \\ Email: dbnjnbanerjee7@gmail.com \\ ${ }^{2}$ Assistant Professor, Department of Mathematics, \\ NIT Durgapur, 713209, West Bengal, India \\ Email: anita.buie@gmail.com
}

\begin{abstract}
A signed graph may be defined as an ordered pair $(G, \sigma)$ where $G=(V, E)$ is the graph and $\sigma$ is a function called signature or sign mapping from $E$ to the sign group $\{+,-\}$. People or friends are considered as the vertices and the relationships between these friends are considered as the edges. ' + ' sign is assigned to the edge for good relationship and '-' sign for bad relationship between the friends. Two terms "friendship matrix" or "judgement matrix" and "character point C.P" are introduced in this paper. Using the concept of friendship matrix and C.P in various fields the characteristics or performances could be evaluated. In this paper different problems have been considered and analysed with the help of signed graph.
\end{abstract}

\section{Indexing terms/Keywords}

Character point, character ratio, friendship matrix, half edge, loose edge, marked signed graph, signed graph, signed pedigree graph.

\section{Academic Discipline And Sub-Disciplines}

Mathematics; Social Psychology; Biology

\section{SUBJECT CLASSIFICATION}

AMS Mathematics Subject Classification(2010) - 97K30

\section{TYPE (METHOD/APPROACH)}

Analysis of several problems

\section{Council for Innovative Research}

Peer Review Research Publishing System

Journal: INTERNATIONAL JOURNAL OF COMPUTERS \& TECHNOLOGY

Vol.14, No.3

www.ijctonline.com, editorijctonline@gmail.com 


\section{INTRODUCTION}

A "signed graph" is associated with two signs ' + ' and '-'. The signature of S, assigns a ' + ' or '-' sign to every edge, accordingly designating it as being either positive or negative. Signed graphs were first introduced by Frank Harary to handle a problem in social psychology along with the psychologist Dorwin Cartwright. In social psychology, signed graphs have been used in social situations where the vertices or the nodes represented people, the positive edges represented friendship and the negative edges represented enmities between nodes.

Basically, a friendship matrix or judgement matrix is an $m \times n$ matrix whose elements can be +1 and -1 only. The matrix should always be associated with character points because a friendship matrix without character points will be considered as invalid. "Character point C.P is a point or score which determines the characteristics or nature of person, place, time, object or action." Generally, the signed graph associated with the matrix is a complete graph. The matrix gives the best result if the number of rows and columns in the matrix are odd because in that case, C.P will never be zero. The disadvantage of C.P is that, no definite conclusion can be drawn if C.P $=0$. The main objective of this paper is to apply the concept of signed graph in decision making.

Definition 1: [1] A signed graph in which all the edges are positive is known as all-positive signed graph and the signed graph in which all the edges are negative is known as all-negative signed graph. A signed graph is said to be homogeneous if it is either all-positive or all-negative and heterogeneous otherwise.

Definition 2: [1] A marked signed graph is an ordered pair $(S, \mu)$ where $S=(G, \sigma)$ is a signed graph and $\mu: V(G) \longrightarrow\{+1,-1\}$ is a function called marking of $S$ from the vertex set $V(G)$ of $G$ to the set $\{+1,-1\}$.

Definition 3: [1] A marked signed graph $S$ is said to be cycle-compatible if for every cycle $Z$ in $S$ the product of the signs of its vertices is equal to the product of the signs of its edges.

Definition 4: [2] The sign of a circle (circle is the edge set of a simple cycle) is defined to be the product of the signs of its edges. A signed graph is said to be balanced if it has no half-edges and every circle in it is positive.

Definition 5: A half-edge is an edge having only one end point.

Definition 6: [1] A graph $G=(V, E)$ together with a function $\mathrm{f}: \mathrm{V} \mathbf{U} \mathrm{E} \longrightarrow\{-1,+1\}$ is called a net, denoted by $N=$ $(G, f)$. A cycle $Z$ in the net $N$ is said to be consistent or inconsistent according to whether its sign is positive or negative. The net $\mathrm{N}$ as a whole is said to be consistent if all its cycles are consistent and inconsistent otherwise.

Definition 7: [1] A signed graph is said to be sign-compatible if its vertices can be marked such that end vertices of an edge in it are both negative if and only if the edge is negative. A signed graph $S$ is sign-compatible if and only if its vertex set $\mathrm{V}(\mathrm{S})$ can be partitioned into two subsets $\mathrm{V} 1$ and $\mathrm{V} 2$ such that the all-negative sub graph of $\mathrm{S}$ is precisely the sub graph induced by exactly one of the subsets V1 and V2.

Definition 8: [4] if $S$ is a signed graph, we define a signed coloring of $S$ in $\mu$ colors or in $2 \mu+1$ colors, to be a mapping

$\mathrm{K}: \mathrm{N} \rightarrow[-\mu, \mu]=\{-\mu+1, \ldots ., 0, \ldots \ldots, \mu-1, \mu\}$

where $\mathrm{N}$ is the node set of $\mathrm{S}$.

Definition 9: [1] a signed graph $S$ is skew-balanced if for every cycle $Z$ in $S$ either $Z$ is homogeneous or the number of negative sections in $Z$ is even.

Now let us explain the concept of signed graphs diagrammatically:


Signed graph $S=(G, \sigma)$

$$
\text { graph } G=(V, E)
$$

Figure 1: diagrammatic representation of signed graph 


\section{APPLICATIONS}

\section{Representation of 'fair' friendship}

The objective of this problem is to determine whether a person is good or bad on the basis of his relationship with other friends. Given below a signed graph where the vertices represent the five friends $A, B, C, D$ and $E$ and the edges represent the mutual relationships among the five friends. Here ' + ' sign represents good relationship and '-' sign represents bad relationship among the two friends. We also see that the graph is a complete graph.

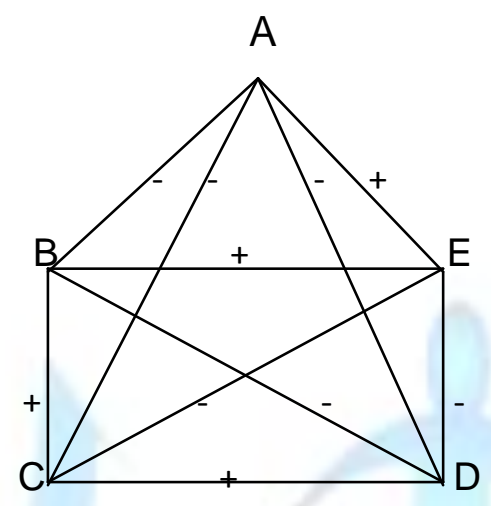

Figure 2: Signed graph showing one-way relationships among 5 friends

Corresponding friendship matrix with C.P is given below, where +1 is given for a good relationship between friends and -1 otherwise.

\begin{tabular}{r|ccccc|c} 
& A & B & C & D & E & character points \\
\hline A & +1 & -1 & -1 & -1 & +1 & -1 \\
B & -1 & +1 & +1 & -1 & +1 & +1 \\
C & -1 & +1 & +1 & +1 & -1 & +1 \\
D & -1 & -1 & +1 & +1 & -1 & -1 \\
E & +1 & +1 & -1 & -1 & +1 & +1 \\
Character & -1 & +1 & +1 & -1 & +1 & Total character point $=+1$ \\
Points & & & & & &
\end{tabular}

Obviously, a person loves himself and so all the diagonal elements here are +1 .

Sum of the elements of the A-row or A-column gives the character point of A and similarly for B, C, D and E.

Total C.P $=$ sum of the C.Ps of the rows or of the columns $=-1+1+1-1+1=+1$.

Here we are trying to determine whether a person is good or bad on the basis of his relationship with other friends, i.e., we make an assumption that if a person has a good relationship with almost all his friends then he is good but if a person has a bad relationship with most of his friends, then he is considered as bad because he must have some negative qualities in him due to which his relationship is bad with most of his friends.

As per our assumption, $B, C$ and $E$ are good persons as they have $C . P+1(>0)$ and $A, D$ are bad as they have $C . P-1(<0)$. Now the total character point tells us about the friend circle or the whole group. Here the total C.P is +1 , i.e., the friend circle or the group A-B-C-D-E is good.

Knowing a person properly is not a very easy job in this world but here we have just made a mathematical attempt to do so. We also note that the matrix obtained here is a symmetric matrix.

\section{Result:}

Thus we can see that, by observing the type of relationship of a person with other persons we can easily determine his nature, in fact, the nature of the whole friend circle with the help of our newly introduced matrix called "friendship matrix" which is based on the concept of signed graph. 


\section{Representation of 'unfair' friendship}

In section 1, we have considered only one-way relationships but now we will consider two-way relationships. The objective of this problem is same as that of section 1. Suppose there are two friends A and B. It may happen that, A likes B but B does not like A. The "friendship matrix" in this case will not be symmetric. Let us consider the following example. Given below is a signed graph, where the edges represent the relationship among two friends and the vertices denote the friends respectively but in this section the graph is a directed graph as shown below:

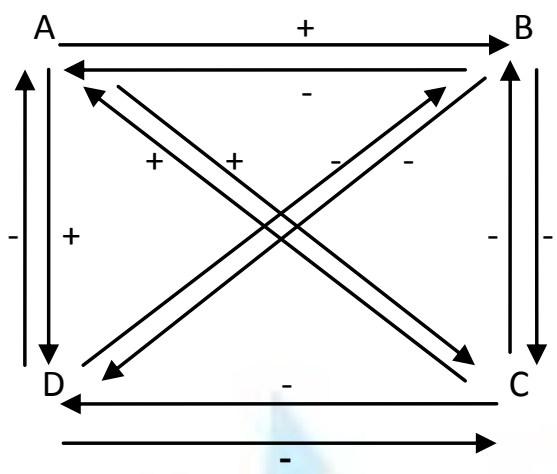

Figure 3: Signed graph showing two-way relationships among 4 friends

The friendship matrix of the above graph is as given below:

\begin{tabular}{c|cccc|c} 
& A & B & C & D & C.P \\
\hline A & +1 & +1 & +1 & +1 & +4 \\
B & -1 & +1 & -1 & -1 & -2 \\
C & +1 & -1 & +1 & -1 & 0 \\
D & -1 & -1 & -1 & +1 & -2 \\
\hline C.P & 0 & 0 & 0 & 0 & Total C.P $=0$
\end{tabular}

Here, the sum of the elements of the A-row gives the character point of $A$ as +4 but as the matrix is not symmetric, sum of the elements of the A-column gives a different character point of $A$ as 0 . This is similar in case of $B, C$ and $D$. So,

C.P of $A=+4+0=+4$ (i.e., sum of the C.P of the row and column of $A$ )

C.P of $B=-2+0=-2$

C.P of $\mathrm{C}=0+0=0$

C.P. of $D=-2+0=-2$.

Total C.P $=$ sum of the C.Ps of the rows or the columns $=+4-2+0-2=0+0+0+0=0$.

So, by our assumption of section $1, A$ is the best person among the four, $B$ and $D$ are worse persons among the four but we cannot say anything about $C$. So we may consider $C$ as a neutral person i.e., neither good nor bad.

Total C.P $=0$. That means the friend circle or the group A-B-C-D is neither good nor bad. This is one disadvantage of character point, i.e., if C.P $=0$, we cannot say anything properly.

\section{Result:}

Thus we can see that, in case of a two-way relationship we are also able to determine the nature of a person from the character points after constructing our friendship matrix. In fact, we are able to determine the nature of the whole group or the friend circle.

\section{Application in the educational field}

The friendship matrix has its application not only in friendships and relationships but it can also be applied in the educational field. For e.g., the marks distribution of some students in certain subjects, performances of schools in a certain region for a particular period of time(say 5 years), etc. These are described below:

Case 1: Let us consider 4 students $P, Q, R$ and $S$ and 3 subjects, say, Physics, Chemistry and Mathematics. Below is given a signed graph where '+' sign represents 'pass' and '-' sign represents 'fail'. 


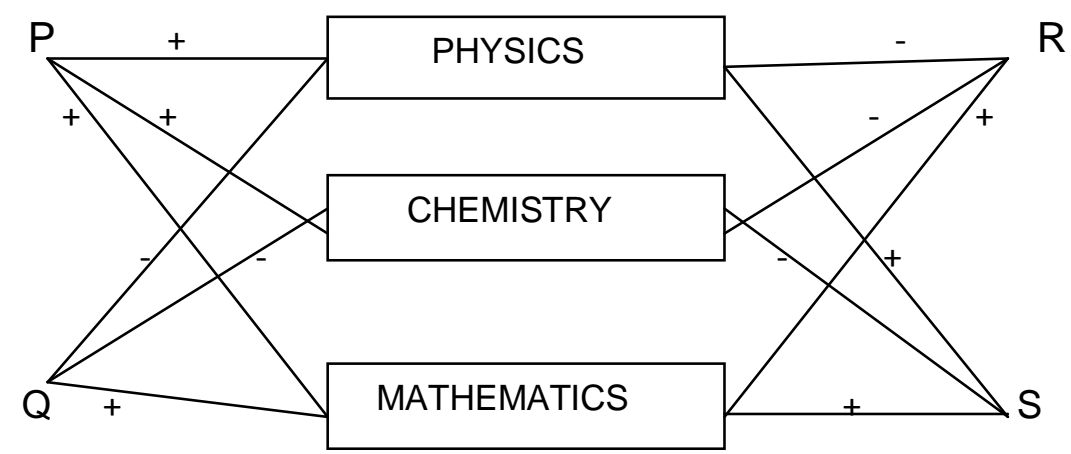

Figure 4: Signed graph showing 'pass' and 'fail' of 4 students $P, Q, R, S$ in 3 subjects

Now we construct the matrix where, +1 is given if a student passes in a certain subject and -1 is given if a student fails.

\begin{tabular}{l|cccc|c} 
& $\mathrm{P}$ & $\mathrm{Q}$ & $\mathrm{R}$ & $\mathrm{S}$ & $\mathrm{C} . \mathrm{P}$ (subject-wise performance) \\
\hline PHYSICS & +1 & -1 & -1 & +1 & 0 \\
CHEMISTRY & +1 & -1 & -1 & -1 & -2 \\
MATHEMATICS & +1 & +1 & +1 & +1 & +4 \\
\hline C.P & +3 & -1 & -1 & +1 & Total C.P $=+2$ \\
(Student-wise) & & & &
\end{tabular}

From the subject-wise performance, we see that performance in Mathematics is the best, performance in Physics is medium and performance in Chemistry is the worst. So special attention should be given towards Chemistry.

From the student-wise performance, we see that performance of $\mathrm{P}$ is the best, $\mathrm{Q}$ and $\mathrm{R}$ give a worse performance and that of $\mathrm{S}$ is more or less good. But the total performance is good because total C.P $=+2$, which is positive.

We also notice that the matrix obtained here is not a square matrix. It's a $3 \times 4$ matrix.

The origin of the 'friendship matrix' is from the field of graph theory (to be more specific: signed graph) and social psychology but the matrix also has its application in the field of Statistics which is discussed in case 2.

Case 2: Suppose in a particular region, there are 4 schools A, B, C and D. The performances of these schools for the past seven years are given below:

Table 1: Pass percentages of different schools from 2008 to 2014

\begin{tabular}{|l|l|l|l|l|l|l|l|}
\hline & & & & & & \\
\hline SCHOOLS & 2008 & 2009 & 2010 & 2011 & 2012 & 2013 & 2014 \\
\hline SCHOOL A & $80 \%$ & $95 \%$ & $70 \%$ & $90 \%$ & $80 \%$ & $96 \%$ & $89 \%$ \\
\hline SCHOOL B & $96 \%$ & $80 \%$ & $97 \%$ & $72 \%$ & $99 \%$ & $88 \%$ & $90 \%$ \\
\hline SCHOOL D & $98 \%$ & $99 \%$ & $100 \%$ & $100 \%$ & $89 \%$ & $88 \%$ & $97 \%$ \\
\hline
\end{tabular}

(Here, '\%' represents the pass percentage of schools in ICSE in a particular year. For e.g., the pass \% for school A in ICSE in the year 2012 is $80 \%$ ).

Our main aim is to judge the schools by their respective performances in the past seven years. We will do this judgement by using our newly introduced 'friendship matrix'. 
We will assign +1 if the pass $\% \geq 90 \%$ and -1 if the pass $\%<90 \%$. The matrix is given as follows:

\begin{tabular}{|c|c|c|c|c|c|c|c|c|}
\hline${ }_{\text {Schools }}^{\text {Years }}$ & 2008 & 2009 & 2010 & 2011 & 2012 & 2013 & 2014 & $\begin{array}{l}\text { C.P (school-wise } \\
\text { performance) }\end{array}$ \\
\hline$A$ & -1 & +1 & -1 & +1 & -1 & +1 & -1 & -1 \\
\hline B & +1 & -1 & +1 & -1 & +1 & -1 & +1 & +1 \\
\hline C & +1 & +1 & +1 & +1 & -1 & -1 & +1 & +3 \\
\hline $\mathrm{D}$ & -1 & -1 & -1 & +1 & -1 & +1 & -1 & -3 \\
\hline $\begin{array}{l}\text { C.P (year-wise } \\
\text { Performance) }\end{array}$ & 0 & 0 & 0 & +2 & -2 & 0 & 0 & Total C.P $=0$ \\
\hline
\end{tabular}

From the above matrix we can see that school $C$ has given its best performance with C.P +3 and school $D$ has performed worst with C.P -3 in the past seven years. Now if we consider the year-wise performances, then in the year 2011 the performance was best with C.P +2 and in the year 2012, the performance was worst with C.P -2 .

Here our judgement is totally based on the assumption that: "if the pass $\%$ of a school $\geq 90 \%$, the school is good and if pass $\%$ of a school $<90 \%$, it is bad." So we will not calculate the average pass $\%$ for each student or each year and compare with the matrix because it may not match.

Thus we can also call a 'friendship matrix' as a 'judgement matrix' because it is used in judgement. We also observe that the matrix in this case has been constructed on the basis of statistical data.

Case 3: Let us consider a student $A$ and his \% of marks in Physics and Statistics in the years 2010-2013 given as follow:

Table 2: Percentages of marks obtained by a student in Physics and Statistics

\begin{tabular}{|l|l|l|l|l|l|}
\hline SUBJECTS & & & & & \\
\hline PHYSICS & 2010 & 2012 & 2013 & AVERAGE $\%$ \\
\hline STATISTICS & $30 \%$ & $90 \%$ & $90 \%$ & $70 \%$ & $70 \%$ \\
\hline
\end{tabular}

We can see that, in both Physics and Statistics his average performance is same since the average \% in both the cases is $70 \%$. So we cannot determine that in which subject he has performed better. Under this situation, the concept of character point in our judgement matrix will be useful.

In the matrix, let us give +1 if $\%$ of marks $\geq 75 \%$ and -1 otherwise as given below:

\begin{tabular}{|c|c|c|c|c|c|}
\hline Subjects & 2010 & 2011 & 012 & 2013 & C.P \\
\hline Physics & -1 & +1 & +1 & -1 & 0 \\
\hline Statistic & -1 & +1 & +1 & +1 & +2 \\
\hline C.P & -2 & +2 & +2 & 0 & Total C.P $=+2$ \\
\hline
\end{tabular}

From the above matrix we see that:

C.P in Statistics $=+2>$ C.P in Physics $=0$. Then obviously, A's performance is better in Statistics. So, one may suggest A to choose Statistics as a subject for higher studies in future by observing his past four years' \% of marks.

\section{Application in industrial problem}

Let us consider 5 machines A, B, C, D and E which can perform 5 jobs independently. The objective of this problem is to determine that which machine is performing badly so that we can replace them by the better ones. The efficiencies of the machines are given in percentage below: 
Table 3: Efficiencies of machines in percentages performing different jobs

\begin{tabular}{c|ccccc} 
& JOB1 & JOB2 & JOB3 & JOB4 & JOB5 \\
\hline A & $70 \%$ & $90 \%$ & $95 \%$ & $96 \%$ & $97 \%$ \\
B & $80 \%$ & $92 \%$ & $85 \%$ & $86 \%$ & $90 \%$ \\
C & $92 \%$ & $79 \%$ & $93 \%$ & $87 \%$ & $97 \%$ \\
D & $93 \%$ & $95 \%$ & $89 \%$ & $82 \%$ & $79 \%$ \\
E & $86 \%$ & $79 \%$ & $72 \%$ & $95 \%$ & $73 \%$
\end{tabular}

Efficiency of machine $=$ (output/input) $\times 100$.

Now we construct the judgement matrix, where +1 is given if efficiency $\geq 85 \%$ and -1 otherwise, as follows

\begin{tabular}{l|lllll|l} 
& Job1 & job2 & job3 & job4 & job5 & C.P \\
\hline A & -1 & +1 & +1 & +1 & +1 & +3 \\
B & -1 & +1 & +1 & +1 & +1 & +3 \\
C & +1 & -1 & +1 & +1 & +1 & +3 \\
D & +1 & +1 & +1 & -1 & -1 & +1 \\
E & +1 & -1 & -1 & +1 & -1 & -1 \\
\hline C.P & +1 & +1 & +3 & +3 & +1 & Total C.P $=+9$
\end{tabular}

\section{Result:}

Machine $E$ has C.P -1 , that means it is the worst machine and should be replaced by a better one. Machines A, B, C are excellent and machine $D$ is good.

\section{Application in the study of spreading of cancer}

In this section we will see how cancer spreads both generation-wise and cell-wise.

\section{Definitions:}

1. Cancer: A group of diseases in which body cells undergo uncontrolled division.

2. Tumour: Mass of cells that grow and divide without any obvious function in the body.

3. Benign tumour: Mass of cells that remain within the tissue in which it originated and do not affect the surrounding tissues.

4. Malignant tumour: Mass of cells that invade the surrounding cells. Here the cells are abnormal in appearance.

\section{Case 1: Generation-Wise Spreading}

Given below is a signed pedigree graph/tree:

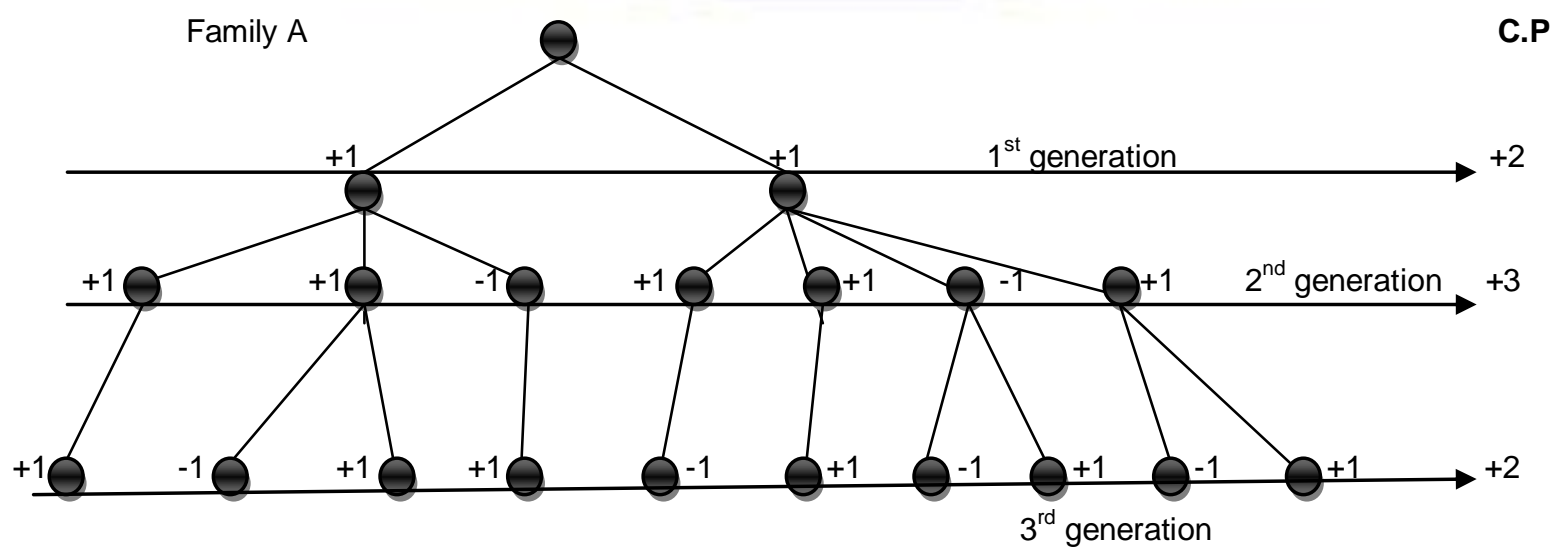

Figure 5: Signed pedigree tree showing the cancer affected patients in generation 1, 2 and 3 
C.P denotes 'character point' as defined earlier. Here the vertices represent the persons in a particular generation. +1 is given if a person is not suffering from cancer and -1 is given if a person is suffering from cancer.

Here, the C.Ps are calculated by adding the respective $+1 \mathrm{~s}$ and $-1 \mathrm{~s}$ in a particular generation.

Now, we will introduce another new concept called 'character ratio', where

Character ratio $(\mathrm{Cr})=$

Character point

No. of persons/observations

Our objective is to determine the chance of a person in a particular generation to suffer from cancer by observing the value of Cr.

For the $1^{\text {st }}$ generation, $\mathrm{Cr}=+2 / 2=+1$ (no cancer patient)

For the 2nd generation, $\mathrm{Cr}=+3 / 7=+0.42$

For the $3^{\text {rd }}$ generation, $\mathrm{Cr}=+2 / 10=+0.2$ (maximum number of cancer patients).

Here we can notice a drawback of C.P. In the $1^{\text {st }}$ generation, C.P is +2 and there is no cancer patient but in the $3^{\text {rd }}$ generation also C.P is +2 and there are several cancer patients. So C.P is not giving a proper judgment here. That's why we introduce the concept of 'character ratio' which gives us a proper judgment. This is due to the fact that, in case of C.P we were not considering the no. of persons or observations but in case of character ratio we are considering it.

Here, we also note that $\mathrm{Cr}$ always lies between -1 and +1 .

$$
\text { i.e., }-1 \leq \mathrm{Cr} \leq+1 \text {. }
$$

In the pedigree signed graph/tree, we are designating each vertex as +1 or -1 . So the graph here is a marked signed graph.

\section{Result:}

Thus we see that, more is the character ratio in a particular generation, less will be the chance of a person in that generation to suffer from cancer.

\section{Case 2: Cell-Wise Spreading}

The graph given below is a signed pedigree graph where the vertices represent body cells. +1 is given if a cell is not cancerous and -1 is given if a cell is cancerous. Three arbitrary cells are picked from the site of origin of cancer and are considered as the first (initial) stage of cell division.

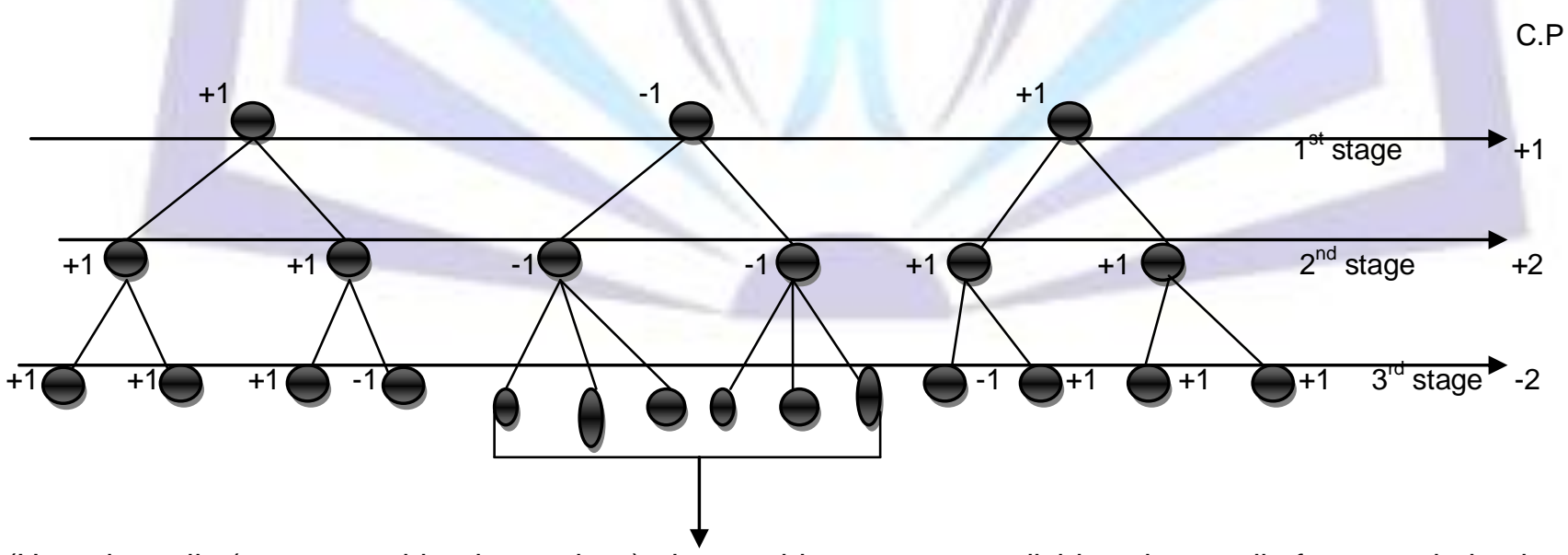

(Here the cells (represented by the vertices) abnormal in appearance divides abnormally faster and also invades the nearby or distant cells. Here all the vertices are marked as -1.)

Figure 6: Signed pedigree graph showing various stages of cancerous cell division

Our objective is to determine the malignant stage by observing the value of $\mathrm{Cr}$.

For the $1^{\text {st }}$ stage, character ratio $(\mathrm{Cr})=+1 / 3$

For the $2^{\text {nd }}$ stage, $\mathrm{Cr}=+2 / 6$ 
For the $3^{\text {rd }}$ stage, $\mathrm{Cr}=-2 / 14$ (malignant stage)

\section{Result:}

In the $3^{\text {rd }}$ stage the $\mathrm{Cr}$ is negative, i.e., this stage is very dangerous. In this stage the cancerous cells also invade the nearby cells and as a result the cancer spreads inside the body increasing the danger. So the $3^{\text {rd }}$ stage is the malignant stage.

\section{Application in the passenger problem}

Suppose R1, R2 and R3 are three routes of approximately the same distance connecting two towns $A$ and $B$. There are three buses $B 1, B 2$ and $B 3$ travelling through these routes $R 1, R 2$ and $R 3$ respectively. A passenger $P$ wants to travel from town $A$ to town $B$ in such a way that it takes minimum time to reach. His convenient day of travel is Sunday, Monday or Tuesday. The graph associated with this problem is given below

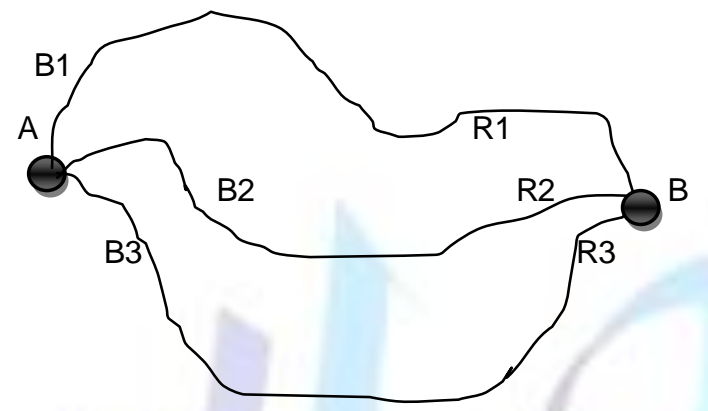

Figure 7: Graph showing three buses B1, B2, B3 travelling through three routes R1, R2, R3

We consider the times of journey of the three buses from $A$ to $B$ in three consecutive days as given below:

Table 4: Journey time of three buses B1, B2, B3 on three consecutive days

\begin{tabular}{|l|l|l|l|}
\hline DAYS & B1 & B2 & B3 \\
\hline SUNDAY & & & \\
\hline MONDAY & 18 mins. & 21 mins. & 19 mins. \\
\hline TUESDAY & 22 mins. & 25 mins. & 24 mins. \\
\hline $\begin{array}{l}\text { AVERAGE } \\
\text { TIME }\end{array}$ & 26 mins. & 20 mins. & 23 mins. \\
\hline
\end{tabular}

We can see that the average times of journey of the three buses are same. As the average times of journey of the three buses are same, so it is difficult for the passenger $\mathrm{P}$ to choose a bus such that his journey time is minimum. We will solve the problem by constructing the judgement matrix, where +1 will be given if the journey time $\leq$ the average time $(22$ mins.) and -1 otherwise, which is given as follows

\begin{tabular}{l|rrr|l} 
Buses & B1 & B2 & B3 & C.P \\
\hline Sunday & +1 & +1 & +1 & +3 \\
Monday & +1 & -1 & -1 & -1 \\
Tuesday & -1 & +1 & -1 & -1 \\
\hline C.P & +1 & +1 & -1 & Total C.P $=+1$
\end{tabular}

The passenger $\mathrm{P}$ can choose any one of bus $\mathrm{B} 1$ or bus $\mathrm{B} 2$ since $\mathrm{C} . \mathrm{P}$ of $\mathrm{B} 1=\mathrm{C} . \mathrm{P}$ of bus $\mathrm{B} 2=+1>0$. 
But as C.P of bus $B 3=-1<0$, so passenger $P$ rejects $B 3$.

On the other hand, the passenger $P$ will always try to go on Sunday since C.P for Sunday $=+3$ (maximum).

\section{Result:}

The passenger $\mathrm{P}$ will choose any one of bus B1 or bus B2 and he will always try to go on Sunday.

\section{CONCLUSION}

Thus we have applied the concept of signed graph in decision-making especially by using the newly introduced concept of 'Character points'. This concept can be applied in miscellaneous fields such as Social Psychology, Statistics, for industrial purposes, in the study of spreading of cancer, in the passenger problem etc. By using this concept, we can point out the negative properties in any person, place, time, object or action and can clarify them. For e.g., in section 2.4 , machine $E$ can be replaced by a better one. We can also take decisions for future purposes. For e.g., in case 3 of section 2.3, student $A$ can choose Statistics for his higher studies in future.

\section{ACKNOWLEDGMENT}

The authors would like to express their gratitude to all the referees for their valuable comments and thanks the members of the editorial board of the journal for publishing the paper.

\section{REFERENCES:}

[1] Belmannu Devadas Acharya, Mukti Acharya and Deepa Sinha, Forcibly cycle-compatible signed line graphs, presented at the ' $39^{\text {th }}$ Annual Conference of the Ramanujan Mathematical society' held at the Institute of Basic Sciences, B.R. Ambedkar University , Agra during July 21-24, 2004.

[2] Thomas Zaslavsky, Signed graphs and geometry, Department of Mathematical Sciences, Binghamton University, Binghamton, NY13902-6000, U.S.A.

[3] Thomas Zaslavsky, Signed graphs, Discrete Applied Mathematics 4(1982) 47-74.

[4] Thomas Zaslavsky, Signed graph coloring, Discrete Mathematics 39(1982) 215-228.

[5] Dorwin Cartwright and Frank Harary, The Psychological Review, Structural balance: a generalization of Heider's theory, VOL. 63, NO. 5, September, 1956.

\section{Author's biography with Photo}

Debanjan Banerjee received his B.Sc. degree in Mathematics from the University of Kalyani. Presently he is a student of M.Sc. Mathematics at National Institute of Technology, Durgapur.

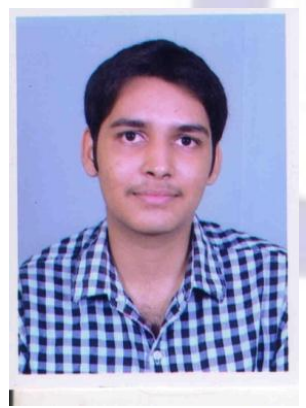

Anita Pal received her Ph.D. degree in Mathematics and has been teaching for more than seven years to B.Tech, M.Sc., M.Tech, students. Presently she is the Assistant Professor of Department of Mathematics in National Institute of Technology, Durgapur. She has published several research papers in many national and international journals. Her current research interest includes: Discrete Mathematics, Graph Theory and Fuzzy Mathematics

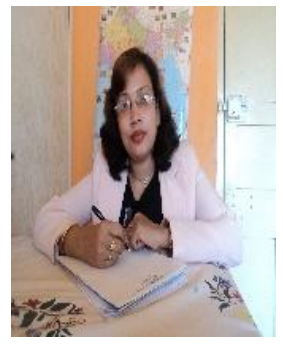

